PROCEEDINGS OF THE

AMERICAN MATHEMATICAL SOCIETY

Volume 137, Number 11, November 2009, Pages 3849-3859

S 0002-9939(09)09963-8

Article electronically published on June 15, 2009

\title{
THOMPSON ISOMETRIES OF THE SPACE OF INVERTIBLE POSITIVE OPERATORS
}

\author{
LAJOS MOLNÁR
}

(Communicated by Nigel J. Kalton)

\begin{abstract}
We determine the structure of bijective isometries of the set of all invertible positive operators on a Hilbert space equipped with the Thompson metric or the Hilbert projective metric.
\end{abstract}

\section{INTRODUCTION AND STATEMENT OF THE RESULTS}

In this paper we consider the Thompson metric and the Hilbert projective metric on the set of all invertible positive operators acting on a Hilbert space. The general approach to these distances is as follows.

Let $E$ be a real normed space and $K$ be a nonempty closed convex cone in $E$ with the property that $K \cap(-K)=\{0\}$. It is well known that $K$ induces a partial order $\leq$ on $E$, namely, we have $x \leq y$ if and only if $y-x \in K$. Clearly, $K=\{x \in E: x \geq 0\}$. Consider the equivalence relation $\sim$ on $K \backslash\{0\}$ given by $x \sim y$ if and only if there exist positive real numbers $r$ and $s$ such that $r x \leq y \leq s x$. We call the corresponding equivalence classes components. The Thompson metric $d_{T}$ on a component $C$ is defined in the following manner. For any $x, y \in C$ write $M(x / y)=\inf \{t>0: x \leq t y\}$ and set

$$
d_{T}(x, y)=\log \max \{M(x / y), M(y / x)\} .
$$

In his paper [19] Thompson introduced this quantity as a modification of the Hilbert projective metric, which is defined on $C$ by the formula

$$
d_{H}(x, y)=\log (M(x / y) M(y / x)) .
$$

In fact, $d_{H}$ is only a pseudo-metric. We have $d_{H}(x, y)=0$ if and only if $x=t y$ for some $t>0$. The origin of this pseudo-metric goes back to D. Hilbert, who, in his paper [10] of 1903 on the foundations of geometry, introduced it for the projective plane. In $1957 \mathrm{G}$. Birkhoff 4 discovered that this kind of distance is a useful tool for finding solutions of some linear integral equations. Later the applications extended to solving a variety of algebraic, integral and differential equations. The modification of $d_{H}$ to $d_{T}$ given by Thompson further widened the range of problems

Received by the editors December 22, 2008, and, in revised form, March 3, 2009, and March 7, 2009.

2000 Mathematics Subject Classification. Primary 46B28, 47B49.

The author was supported by the Hungarian National Foundation for Scientific Research (OTKA), Grant No. NK68040. 
to which these methods were applicable. A modern treatment of the topic can be found in the monograph [15.

Thompson proved in [19] that $d_{T}$ is a true metric on each component which is furthermore complete provided that the cone $K$ is norm complete and normal, i.e., there exists a positive real number $\gamma$ such that $\|x\| \leq \gamma\|y\|$ holds whenever $0 \leq x \leq y$.

It is easy to see that for any unital $C^{*}$-algebra $\mathcal{A}$, the cone $\mathcal{A}^{+}$of all positive elements is a closed normal cone and the set $\mathcal{A}_{-1}^{+}$of all invertible positive elements of $\mathcal{A}$ is a component. It was proved in 3 that on this component, $d_{T}$ coincides with the geodesic distance corresponding to the natural Finsler geometrical structure of $\mathcal{A}_{-1}^{+}$. Hence, there is a close link between the Thompson metric and the differential geometry of $\mathcal{A}_{-1}^{+}$. At this point we recall that the differential geometry of the space of $n \times n$ positive definite complex matrices is an important research field because of its applications to problems coming from many different areas, the most important of which concern linear systems, statistics, filters, Lagrangian geometry and quantum systems (see [5]).

The aim of this paper is to determine the structure of the bijective isometries of the set of all invertible positive operators acting on a Hilbert space equipped with the Thompson metric or the Hilbert projective metric. The study of isometries of spaces of functions and operators is a rather extensive research area with the most classical results being the Banach-Stone theorem and Kadison's description of the bijective linear isometries of $C^{*}$-algebras. For a recent monograph on the topic we refer to the two volume set [6, 7].

In what follows, let $H$ be a complex Hilbert space and denote by $B(H)$ the $C^{*}$-algebra of all bounded linear operators on $H$. It is not difficult to see that the Thompson metric on $B(H)_{-1}^{+}$is given by

$$
d_{T}(A, B)=\left\|\log A^{-1 / 2} B A^{-1 / 2}\right\|
$$

for any $A, B \in B(H)_{-1}^{+}$. A proof of this fact can be found, for example, in [3, Proposition], but it also follows from the discussion below. Concerning the Hilbert projective metric, we have

$$
d_{H}(A, B)=\operatorname{diam} \log \left(\sigma\left(A^{-1 / 2} B A^{-1 / 2}\right)\right)
$$

for all $A, B \in B(H)_{-1}^{+}$. To see this, we compute the quantity $M(A / B)$. For any real number $t>0$ we have

$$
\begin{aligned}
A \leq t B \Leftrightarrow & \frac{1}{t} I \leq A^{-1 / 2} B A^{-1 / 2} \Leftrightarrow \sigma\left(A^{-1 / 2} B A^{-1 / 2}\right) \subset[1 / t, \infty[ \\
& \left.\left.\Leftrightarrow-\log \left(\sigma\left(A^{-1 / 2} B A^{-1 / 2}\right)\right) \subset\right]-\infty, \log t\right] .
\end{aligned}
$$

(Here and below $\sigma($.$) stands for the spectrum.) Therefore, we obtain that$

$$
\begin{gathered}
\log M(A / B)=\max \left(-\log \left(\sigma\left(A^{-1 / 2} B A^{-1 / 2}\right)\right)\right) \\
=-\min \log \left(\sigma\left(A^{-1 / 2} B A^{-1 / 2}\right)\right) .
\end{gathered}
$$

In a similar way one can check that $M(B / A)=\max \log \left(\sigma\left(A^{-1 / 2} B A^{-1 / 2}\right)\right)$ and then conclude that

$$
\begin{gathered}
d_{H}(A, B)=\log M(A / B)+\log M(B / A) \\
=\max \log \left(\sigma\left(A^{-1 / 2} B A^{-1 / 2}\right)\right)-\min \log \left(\sigma\left(A^{-1 / 2} B A^{-1 / 2}\right)\right) \\
=\operatorname{diam} \log \left(\sigma\left(A^{-1 / 2} B A^{-1 / 2}\right)\right) .
\end{gathered}
$$


If one considers the original definitions of the Thompson metric and the Hilbert projective metric given in (11) and (2), it is rather easy to see that for any invertible bounded linear or conjugate-linear operator $T$ on $H$ and any scalar-valued function $\left.\tau: B(H)_{-1}^{+} \rightarrow\right] 0, \infty[$, the transformations

$$
A \longmapsto T A T^{*}, \quad A \longmapsto T A^{-1} T^{*}
$$

and

$$
A \longmapsto \tau(A) T A T^{*}, \quad A \longmapsto \tau(A) T A^{-1} T^{*}
$$

are isometries with respect to $d_{T}$ and $d_{H}$, respectively. Our results show that the reverse statements are also true; there are no other kinds of bijective isometries corresponding to those distances.

Theorem 1. Suppose that $H$ is a complex Hilbert space with $\operatorname{dim} H \geq 3$. Let $\phi: B(H)_{-1}^{+} \rightarrow B(H)_{-1}^{+}$be a bijective isometry with respect to the Thompson metric, i.e., assume that $\phi$ is a bijective map satisfying

$$
d_{T}(\phi(A), \phi(B))=d_{T}(A, B) \quad\left(A, B \in B(H)_{-1}^{+}\right) .
$$

Then $\phi$ is either of the form

$$
\phi(A)=T A T^{*} \quad\left(A \in B(H)_{-1}^{+}\right)
$$

or of the form

$$
\phi(A)=T A^{-1} T^{*} \quad\left(A \in B(H)_{-1}^{+}\right),
$$

where $T$ is an invertible bounded linear or conjugate-linear operator on $H$.

The result concerning the isometries of the Hilbert projective metric reads as follows.

Theorem 2. Let $H$ be as above and suppose that $\phi: B(H)_{-1}^{+} \rightarrow B(H)_{-1}^{+}$is a bijective isometry with respect to the Hilbert projective metric; i.e., $\phi$ is a bijective map satisfying

$$
d_{H}(\phi(A), \phi(B))=d_{H}(A, B) \quad\left(A, B \in B(H)_{-1}^{+}\right) .
$$

Then $\phi$ is either of the form

$$
\phi(A)=\tau(A) T A T^{*} \quad\left(A \in B(H)_{-1}^{+}\right)
$$

or of the form

$$
\phi(A)=\tau(A) T A^{-1} T^{*} \quad\left(A \in B(H)_{-1}^{+}\right),
$$

where $T$ is an invertible bounded linear or conjugate-linear operator on $H$ and $\left.\tau: B(H)_{-1}^{+} \rightarrow\right] 0, \infty[$ is a scalar-valued function.

\section{Proofs}

This section is devoted to the proofs of the results. First, we recall the notion of the geometric mean $A \# B$ of positive operators $A, B \in B(H)^{+}$. This was originally introduced by Pusz and Woronowicz in 18 and later studied in more detail by Ando and several other researchers. See [1]; as a few other relevant papers we also mention [2, 8, 11, 17]. For invertible positive operators $A$ and $B$ we have

$$
A \# B=A^{1 / 2}\left(A^{-1 / 2} B A^{-1 / 2}\right)^{1 / 2} A^{1 / 2} .
$$


This concept will play a crucial role in the proofs of our results. Indeed, the main step in the proof of Theorem 1 is to show that a bijective isometry with respect to the Thompson metric necessarily preserves the geometric mean in the sense that

$$
\phi(A \# B)=\phi(A) \# \phi(B)
$$

holds for all $A, B \in B(H)_{-1}^{+}$. This is in some sense the multiplicative analogue of the celebrated Mazur-Ulam theorem, which asserts that the bijective isometries between real normed spaces preserve the arithmetic mean, implying that these maps are in fact affine. (We remark that the geometric mean preserving bijections of the set $B(H)^{+}$of all positive operators - not of the invertible ones we consider here - have recently been described in [13.)

In the paper [20] Väisälä presented a short ingenious proof of the Mazur-Ulam theorem that was motivated by earlier considerations due to Vogt 21]. That argument gives us the following lemma, which is of fundamental importance for the present results.

Lemma. Let $(M, d)$ be a metric space. Suppose that for each pair $(a, b) \in M \times M$ there exist an element $z(a, b) \in M$, a bijective isometry $\psi_{(a, b)}: M \rightarrow M$ and a constant $k(a, b)>1$ such that

(i) $\psi_{(a, b)}(a)=b, \psi_{(a, b)}(b)=a$;

(ii) $\psi_{(a, b)}(z(a, b))=z(a, b)$;

(iii) $d\left(\psi_{(a, b)}(x), x\right) \geq k(a, b) d(x, z(a, b))$ for all $x \in M$.

If $\phi: M \rightarrow M$ is a bijective isometry, then

$$
\phi(z(a, b))=z(\phi(a), \phi(b))
$$

holds for all $a, b \in M$.

Proof. Consider a fixed pair $(a, b)$ of points in $M$. For convenience, temporarily we write $z, \psi, k$ for $z(a, b), \psi_{(a, b)}, k(a, b)$, respectively. Define

$$
\mathcal{W}=\{\rho: \rho \text { is a bijective isometry of } M, \rho(a)=a, \rho(b)=b\} .
$$

We shall first prove that $\rho(z)=z$ holds for every $\rho \in \mathcal{W}$. To this end, define

$$
c=\sup \{d(\rho(z), z): \rho \in \mathcal{W}\} .
$$

For any $\rho \in \mathcal{W}$ we compute

$$
d(\rho(z), a)=d(\rho(z), \rho(a))=d(z, a),
$$

and hence by the triangle inequality we obtain that

$$
d(\rho(z), z) \leq d(\rho(z), a)+d(a, z)=2 d(z, a) .
$$

This implies that $c$ is finite. Let $\rho \in \mathcal{W}$ be arbitrary as above and define $\tilde{\rho}=$ $\psi \circ \rho^{-1} \circ \psi \circ \rho$. Clearly, $\tilde{\rho}$ is a bijective isometry and, as $\rho$ fixes $a$ and $b$ while $\psi$ interchanges them, it follows that $\tilde{\rho} \in \mathcal{W}$. By (iii) and (ii) we have

$$
\begin{gathered}
k d(\rho(z), z) \leq d(\psi(\rho(z)), \rho(z)) \\
=d\left(\left(\rho^{-1} \circ \psi \circ \rho\right)(z), z\right)=d\left(\left(\psi \circ \rho^{-1} \circ \psi \circ \rho\right)(z), z\right) \\
=d(\tilde{\rho}(z), z) \leq c .
\end{gathered}
$$

Since $\rho \in \mathcal{W}$ was arbitrary, it follows that $k c \leq c$. As $k>1$, we deduce that $c=0$. This proves that $\rho(z)=z$ holds for every $\rho \in \mathcal{W}$. 
Now, let $\phi: M \rightarrow M$ be a bijective isometry. Consider $a, b \in M$ as above and set $a^{\prime}=\phi(a), b^{\prime}=\phi(b)$. Define $\delta=\psi_{(a, b)} \circ \phi^{-1} \circ \psi_{\left(a^{\prime}, b^{\prime}\right)} \circ \phi$. It is easy to check that $\delta \in \mathcal{W}$. We have already learnt that this implies $\delta(z)=z$. From the equality

$$
\left(\psi_{(a, b)} \circ \phi^{-1} \circ \psi_{\left(a^{\prime}, b^{\prime}\right)} \circ \phi\right)(z)=z,
$$

by (ii) we deduce first that $\left(\phi^{-1} \circ \psi_{\left(a^{\prime}, b^{\prime}\right)} \circ \phi\right)(z)=\psi_{(a, b)}^{-1}(z)=z$ and next that $\psi_{\left(a^{\prime}, b^{\prime}\right)}(\phi(z))=\phi(z)$. By (iii) the unique fixed point of $\psi_{\left(a^{\prime}, b^{\prime}\right)}$ is $z\left(a^{\prime}, b^{\prime}\right)$. Therefore, we obtain

$$
\phi(z(a, b))=z(\phi(a), \phi(b)) .
$$

This completes the proof of the lemma.

Before giving the proof of Theorem 1 we make some observations. We first recall the well known fact that for arbitrary elements $x$ and $y$ in a unital algebra we have $\sigma(x y) \backslash\{0\}=\sigma(y x) \backslash\{0\}$. If, in particular, $x$ and $y$ are invertible, then we get $\sigma(x y)=\sigma(y x)$. Next, for all $A, B \in B(H)_{-1}^{+}$we have

$$
\begin{gathered}
d_{T}(A, B)=\left\|\log \left(A^{-1 / 2} B A^{-1 / 2}\right)\right\|=\max \left\{|\lambda|: \lambda \in \sigma\left(\log \left(A^{-1 / 2} B A^{-1 / 2}\right)\right)\right\} \\
=\max \left\{|\lambda|: \lambda \in \log \left(\sigma\left(A^{-1 / 2} B A^{-1 / 2}\right)\right)\right\} \\
=\max \left\{|\lambda|: \lambda \in \log \left(\sigma\left(A^{-1} B\right)\right)\right\},
\end{gathered}
$$

where we have used the spectral mapping theorem and the fact that the norm and the spectral radius of a self-adjoint operator coincide.

Proof of Theorem 1. We shall apply the Lemma in a certain particular case.

To work this out, first pick an arbitrary operator $Z \in B(H)_{-1}^{+}$. We call the mapping $\psi: B(H)_{-1}^{+} \rightarrow B(H)_{-1}^{+}$defined by

$$
\psi(X)=Z X^{-1} Z \quad\left(X \in B(H)_{-1}^{+}\right)
$$

the reflection with respect to $Z$. We verify certain relevant properties of $\psi$. First, it is easy to check that $\psi \circ \psi$ is the identity on $B(H)_{-1}^{+}$. In particular, we obtain that $\psi$ is a bijective map of $B(H)_{-1}^{+}$. Next observe that $\psi$ is an isometry with respect to $d_{T}$. This follows from the remark on the isometric property of transformations of the form $A \mapsto T A^{-1} T^{*}$ that was given in the introduction. Clearly, $Z$ is a fixed point of $\psi$. Moreover, from the equalities

$$
\psi(X)^{-1} X=Z^{-1} X Z^{-1} X=\left(Z^{-1} X\right)^{2}
$$

we obtain

$$
\log \left(\sigma\left(\psi(X)^{-1} X\right)\right)=2 \log \sigma\left(Z^{-1} X\right)
$$

and by (3) this implies that

$$
d_{T}(\psi(X), X)=2 d_{T}(Z, X)=2 d_{T}(X, Z)
$$

for every $X \in B(H)_{-1}^{+}$.

Now, let $A, B \in B(H)_{-1}^{+}$and write $Z=A \# B$. We compute

$$
\begin{gathered}
\psi(A)=Z A^{-1} Z=(A \# B) A^{-1}(A \# B) \\
=\left(A^{1 / 2}\left(A^{-1 / 2} B A^{-1 / 2}\right)^{1 / 2} A^{1 / 2}\right) A^{-1}\left(A^{1 / 2}\left(A^{-1 / 2} B A^{-1 / 2}\right)^{1 / 2} A^{1 / 2}\right) \\
=A^{1 / 2}\left(A^{-1 / 2} B A^{-1 / 2}\right) A^{1 / 2}=B .
\end{gathered}
$$

In a similar manner, using $A \# B=B \# A$ (see Corollary I.2.1 in [1]), one can check that $\psi(B)=A$. 
Consider the Thompson metric $d_{T}$ on $B(H)_{-1}^{+}$. For every pair $(A, B)$ of elements in $B(H)_{-1}^{+}$define $z(A, B)=A \# B$, let $\psi_{(A, B)}$ be the reflection with respect to $A \# B$ and set $k(A, B)=2$. It follows from the above observations that the Lemma applies. Specifically, for any given bijective isometry $\phi: B(H)_{-1}^{+} \rightarrow B(H)_{-1}^{+}$we have that $\phi$ preserves the geometric mean; i.e.,

$$
\phi(A \# B)=\phi(A) \# \phi(B)
$$

holds for all $A, B \in B(H)_{-1}^{+}$. Considering the map

$$
A \longmapsto \phi(I)^{-1 / 2} \phi(A) \phi(I)^{-1 / 2}
$$

we obtain a bijective isometry of $B(H)_{-1}^{+}$which has the additional property that it sends $I$ to itself. In fact, this follows from the observation that for any invertible bounded linear or conjugate-linear operator $T$ on $H$, the map $A \mapsto T A T^{*}$ is a bijective isometry (see the introduction). Therefore, without serious loss of generality, we can assume that the original transformation $\phi$ already has this property; i.e., we have $\phi(I)=I$.

The inverse of an operator $A \in B(H)_{-1}^{+}$can be characterized as the unique solution of the equality $A \# X=I$. Using this characterization, from (5) it follows easily that $\phi\left(A^{-1}\right)=\phi(A)^{-1}$. Moreover, from $A \# I=A^{1 / 2}$ we deduce that

$$
\phi(A)^{1 / 2}=\phi(A) \# I=\phi(A) \# \phi(I)=\phi(A \# I)=\phi\left(A^{1 / 2}\right) .
$$

Pick arbitrary $X, A \in B(H)_{-1}^{+}$and define

$$
B=A^{-1 / 2}\left(A^{1 / 2} X A^{1 / 2}\right)^{2} A^{-1 / 2} .
$$

An easy computation shows that $B=X A X$ and $X=A^{-1} \# B$. Therefore, we have

$$
\phi(X)=\phi\left(A^{-1}\right) \# \phi(B)=\phi(A)^{-1} \# \phi(B),
$$

which gives us that

$$
\phi(X)=\phi(A)^{-1 / 2}\left(\phi(A)^{1 / 2} \phi(B) \phi(A)^{1 / 2}\right)^{1 / 2} \phi(A)^{-1 / 2} .
$$

Multiplying by $\phi(A)^{1 / 2}$ from the left and from the right and squaring, we get

$$
\phi(A)^{1 / 2} \phi(X) \phi(A) \phi(X) \phi(A)^{1 / 2}=\phi(A)^{1 / 2} \phi(B) \phi(A)^{1 / 2} .
$$

This implies that

$$
\phi(X) \phi(A) \phi(X)=\phi(B)=\phi(X A X)
$$

holds for all $X, A \in B(H)_{-1}^{+}$, which means that $\phi$ is a so-called Jordan triple map of $B(H)_{-1}^{+}$. It is not difficult to verify that the norm convergence and the convergence in the Thompson metric coincide on $B(H)_{-1}^{+}$and hence that they induce the same topology (see, e.g., 16, Proposition 1.1]). Therefore, the map $\phi$ is a norm-continuous Jordan triple bijection of $B(H)_{-1}^{+}$. The structure of such transformations was completely described in 12 . Theorem 1 there says that in the infinite dimensional case $\phi$ is either of the form

$$
\phi(A)=U A U^{*} \quad\left(A \in B(H)_{-1}^{+}\right)
$$

or of the form

$$
\phi(A)=U A^{-1} U^{*} \quad\left(A \in B(H)_{-1}^{+}\right),
$$

where $U$ is either a unitary or an antiunitary operator on $H$. Furthermore, it also states that in the case where $3 \leq \operatorname{dim} H<\infty$, the map $\phi$ is either of the form

$$
\phi(A)=(\operatorname{det} A)^{c} U A U^{*} \quad\left(A \in B(H)_{-1}^{+}\right)
$$


or of the form

$$
\phi(A)=(\operatorname{det} A)^{c} U A^{-1} U^{*} \quad\left(A \in B(H)_{-1}^{+}\right),
$$

where $U$ is as above and $c$ is a real number such that $c \neq-1 / \operatorname{dim} H$ in (6) and $c \neq 1 / \operatorname{dim} H$ in (7). It requires only elementary computations to verify that in the finite dimensional case the appearance of the determinant in (6) and (77) can be ruled out (i.e., we have $c=0$ ) as a consequence of the isometric property of $\phi$ with respect to the Thompson metric.

Remembering the reduction $\phi(I)=I$ that we applied in the course of the proof, we can now see that the original map is of one of the forms appearing in the formulation of the theorem.

For the proof of Theorem 2 we recall the general way of defining a true metric on a quotient space of a pseudo-metric space, namely, that the quotient space corresponds to an equivalence relation between the elements of the underlying space. Any two elements are in this relation if they are a zero distance from each other. In the case of the Hilbert projective metric on $B(H)_{-1}^{+}$this means the following. For an arbitrary element $A \in B(H)_{-1}^{+}$the equivalence class $\bar{A}$ is the set of all positive scalar multiples of $A$. Defining $\overline{d_{H}}(\bar{A}, \bar{B})=d_{H}(A, B)$ we obtain a metric on the space of all classes.

Proof of Theorem 2. Let $\phi: B(H)_{-1}^{+} \rightarrow B(H)_{-1}^{+}$be a bijective map which satisfies

$$
d_{H}(\phi(A), \phi(B))=d_{H}(A, B) \quad\left(A, B \in B(H)_{-1}^{+}\right) .
$$

First, we note that the transformation

$$
A \longmapsto \phi(I)^{-1 / 2} \phi(A) \phi(I)^{-1 / 2}
$$

is a bijective map on $B(H)_{-1}^{+}$which preserves the Hilbert projective distance and has the additional property that it maps $I$ to $I$. Indeed, this follows from the observation that for any invertible bounded linear or conjugate-linear operator $T$ on $H$, the map $A \mapsto T A T^{*}$ is a bijective isometry (see the introduction). Therefore, there is no serious loss of generality in assuming that $\phi$ already has the property that $\phi(I)=I$.

It is clear that by defining $\bar{\phi}(\bar{A})=\overline{\phi(A)}\left(A \in B(H)_{-1}^{+}\right)$we obtain a bijective isometry $\bar{\phi}$ on the metric space of all classes $\bar{A}$. Now, one can apply an argument for $\bar{\phi}$ that is very similar to the one we followed in the proof of the first theorem. Specifically, by employing the Lemma one can verify first that $\bar{\phi}$ respects the geometric mean in the sense that

$$
\overline{\phi(A \# B)}=\overline{\phi(A) \# \phi(B)} \quad\left(A, B \in B(H)_{-1}^{+}\right)
$$

and then that $\bar{\phi}$ is a Jordan triple map in the sense that

$$
\overline{\phi(A) \phi(B) \phi(A)}=\overline{\phi(A B A)} \quad\left(A, B \in B(H)_{-1}^{+}\right) .
$$

Unfortunately, unlike in the case of Theorem 1 we cannot continue by using [12, Theorem 1] directly, but we can use the main ideas from its proof. First, upon inserting $B=I$ into (8) we deduce that $\overline{\phi(A)^{2}}=\overline{\phi\left(A^{2}\right)}$ and hence that $\overline{\phi\left(A^{1 / 2}\right)}=$ $\overline{\phi(A)^{1 / 2}}$ holds for all $A \in B(H)_{-1}^{+}$. Therefore, we have

$$
\overline{\phi(A)^{1 / 2} \phi(B) \phi(A)^{1 / 2}}=\overline{\phi\left(A^{1 / 2} B A^{1 / 2}\right)} \quad\left(A, B \in B(H)_{-1}^{+}\right) .
$$


We now prove that $\phi$ preserves commutativity in both directions. This means that for any $A, B \in B(H)_{-1}^{+}$we have $A B=B A$ if and only if $\phi(A) \phi(B)=$ $\phi(B) \phi(A)$. Indeed, it follows from [9, Corollary 3] that for arbitrary positive operators $A$ and $B$ on $H$ we have

$$
A^{1 / 2} B A^{1 / 2}=B^{1 / 2} A B^{1 / 2} \Longleftrightarrow A B=B A .
$$

Let $A, B \in B(H)_{-1}^{+}$. It is easy to see that the above equivalence can be strengthened slightly to the assertion that

$$
A B=B A
$$

if and only if

$$
A^{1 / 2} B A^{1 / 2}=\lambda B^{1 / 2} A B^{1 / 2}
$$

holds for some positive real number $\lambda$. To verify this, suppose that the last equality holds. As we have

$$
\sigma\left(A^{1 / 2} B A^{1 / 2}\right)=\sigma(A B)=\sigma\left(B^{1 / 2} A B^{1 / 2}\right),
$$

it follows that the number $\lambda$ above is necessarily 1 , and hence by the aforementioned result in [9] we obtain $A B=B A$. The reverse implication is obvious. Now, upon applying (9) we see that $\phi$ really preserves commutativity in both directions. Moreover, observe that for any commuting $A, B \in B(H)_{-1}^{+}$we have

$$
\overline{\phi(A B)}=\overline{\phi(A) \phi(B)} \text {. }
$$

Just as in the proof of [12, Theorem 1], using the continuous function calculus, we can define a map $\Phi$ on the set $B_{s}(H)$ of all self-adjoint operators on $H$ by

$$
\Phi(S)=\log \phi\left(e^{S}\right) \quad\left(S \in B_{s}(H)\right) .
$$

It is easy to check that $\Phi$ is a bijective map of $B_{s}(H)$ which preserves commutativity in both directions. Such transformations of $B_{s}(H)$ have recently been characterized in our paper [14. Applying [14, Corollary 2] we obtain that there exists either a unitary or an antiunitary operator $U$ on $H$ and that for every $S \in B_{s}(H)$ there is a real-valued bounded Borel function $g_{S}$ on the spectrum $\sigma(S)$ of $S$ such that

$$
\Phi(S)=U g_{S}(S) U^{*} .
$$

Going back to $\phi$, it is now obvious that for every $A \in B(H)_{-1}^{+}$there is a real-valued bounded Borel function $f_{A}$ on $\sigma(A)$ such that

$$
\phi(A)=U f_{A}(A) U^{*} .
$$

As the transformation $A \mapsto U^{*} \phi(A) U$ is clearly a bijective map on $B(H)_{-1}^{+}$preserving the Hilbert projective distance, we may assume without serious loss of generality that the unitary or antiunitary operator $U$ above is $I$.

So, we have the property of $\phi$ that for every $A \in B(H)_{-1}^{+}$, the operator $\phi(A)$ is a real-valued bounded Borel function of $A$. This implies the following. For any fixed nontrivial projection $P$ on $H$ we have functions $\left.h_{P}, k_{P}:\right] 0, \infty[\rightarrow] 0, \infty[$ such that

$$
\phi\left(\lambda P+P^{\perp}\right)=h_{P}(\lambda) P+k_{P}(\lambda) P^{\perp} .
$$

Picking $0<\lambda, \mu \in \mathbb{R}$ and using the property of $\phi$ that it preserves the Hilbert projective distance, we have

$$
|\log (\lambda / \mu)-\log 1|=\left|\log \left(h_{P}(\lambda) / h_{P}(\mu)\right)-\log \left(k_{P}(\lambda) / k_{P}(\mu)\right)\right|
$$

or, equivalently,

$$
|\log \lambda-\log \mu|=\left|\log \left(h_{P}(\lambda) / k_{P}(\lambda)\right)-\log \left(h_{P}(\mu) / k_{P}(\mu)\right)\right| .
$$


Writing $l=h_{P} / k_{P}$, we obtain

$$
|\log \lambda-\log \mu|=|\log l(\lambda)-\log l(\mu)| .
$$

Considering the function $i: x \mapsto \log l(\exp (x))$ on the real line, we have

$$
|x-y|=|i(x)-i(y)| \quad(x, y \in \mathbb{R}),
$$

which means that $i$ is an isometry of $\mathbb{R}$. It follows easily that $i$ is either of the form $i(x)=x+d$ or of the form $i(x)=-x+d$ with some constant $d \in \mathbb{R}$. Going back to $l$, it follows that it is either of the form $l(\lambda)=c \lambda$ or of the form $l(\lambda)=c / \lambda$ with some positive constant $c$. As $I=\phi(I)=\phi\left(P+P^{\perp}\right)=h_{P}(1) P+k_{P}(1) P^{\perp}$ implies $l(1)=1$, it follows that the constant $c$ is in fact 1 . Therefore, either we have $h_{P}(\lambda) / k_{P}(\lambda)=\lambda$ or we have $h_{P}(\lambda) / k_{P}(\lambda)=1 / \lambda$ holding for all $\lambda>0$.

The above argument verifies that for any chosen nontrivial projection $P$ on $H$ there are two cases, namely, either we have

$$
\overline{\phi\left(\lambda P+P^{\perp}\right)}=\overline{\lambda P+P^{\perp}} \quad(0<\lambda \in \mathbb{R})
$$

or we have

$$
\overline{\phi\left(\lambda P+P^{\perp}\right)}=\overline{\frac{1}{\lambda} P+P^{\perp}} \quad(0<\lambda \in \mathbb{R}) .
$$

We intend to show that the above possibilities in fact do not depend on the particular choice of $P$; i.e., we have either (a) for all nontrivial projections $P$ or (b) for all nontrivial projections $P$. To verify this, first suppose that $P$ is a rank-one projection on $H$ of type (a). Let $Q$ be an arbitrary nontrivial projection on $H$ such that $P \leq Q, P \neq Q$. Suppose that $Q$ is of type (b). It is easy to compute that for any $0<\lambda, \mu \in \mathbb{R}$, the Hilbert projective distance between $\lambda P+P^{\perp}$ and $\mu Q+Q^{\perp}$ is the diameter of the set $\{\log \mu-\log \lambda, \log \mu, 0\}$ while the distance between $\phi\left(\lambda P+P^{\perp}\right)$ and $\phi\left(\mu Q+Q^{\perp}\right)$ is the diameter of the set $\{-\log \lambda-\log \mu,-\log \mu, 0\}$. It is apparent that these two quantities do not coincide for all $0<\lambda, \mu \in \mathbb{R}$, which is a contradiction. Consequently, for any nontrivial projection $Q$ with $P \leq Q$ we have that it is also of type (a). One can show in a similar manner that the property of being of type (b) also "descends upward". It now follows easily that every rank-one projection is of type (a) and then that every nontrivial projection is of type (a), too. In the same way one can verify that if there is a rank-one projection of type (b), then every nontrivial projection is of type (b), too.

Suppose that for every nontrivial projection $P$ on $H$ we have

$$
\overline{\phi\left(\lambda P+P^{\perp}\right)}=\overline{\lambda P+P^{\perp}} \quad(0<\lambda \in \mathbb{R}) .
$$

Let $P_{1}, P_{2}, \ldots, P_{n}$ be pairwise orthogonal nonzero projections on $H$ with sum $I$ and let $\lambda_{1}, \lambda_{2}, \ldots, \lambda_{n}$ be positive real numbers. Set $A=\lambda_{1} P_{1}+\ldots+\lambda_{n} P_{n}$. Using the multiplicativity property (10) of $\phi$ on commuting operators, we compute

$$
\begin{gathered}
\overline{\phi(A)}=\overline{\phi\left(\lambda_{1} P_{1}+\ldots+\lambda_{n} P_{n}\right)}=\overline{\phi\left(\left(\lambda_{1} P_{1}+P_{1}^{\perp}\right) \cdots\left(\lambda_{n} P_{n}+P_{n}^{\perp}\right)\right)} \\
=\overline{\phi\left(\lambda_{1} P_{1}+P_{1}^{\perp}\right) \cdots \phi\left(\lambda_{n} P_{n}+P_{n}^{\perp}\right)}=\overline{\left(\lambda_{1} P_{1}+P_{1}^{\perp}\right) \cdots\left(\lambda_{n} P_{n}+P_{n}^{\perp}\right)} \\
=\overline{\lambda_{1} P_{1}+\ldots+\lambda_{n} P_{n}}=\bar{A} .
\end{gathered}
$$

Therefore, we have that $\overline{\phi(A)}=\bar{A}$ holds for every operator $A \in B(H)_{-1}^{+}$with finite spectrum. Now, let $A \in B(H)_{-1}^{+}$be arbitrary and pick a sequence $A_{n} \in B(H)_{-1}^{+}$ of operators with finite spectrum such that $A_{n} \rightarrow A$ in the operator norm (the 
existence of such a sequence follows from the spectral theorem). By the upper semi-continuity of the spectrum we infer that

$$
d_{H}\left(\phi(A), \phi\left(A_{n}\right)\right)=d_{H}\left(A, A_{n}\right) \rightarrow 0 .
$$

On the other hand, as $\overline{\phi\left(A_{n}\right)}=\bar{A}_{n}$ holds for every $n \in \mathbb{N}$; by the same reason we also obtain

$$
d_{H}\left(\phi(A), \phi\left(A_{n}\right)\right)=d_{H}\left(\phi(A), A_{n}\right) \rightarrow d_{H}(\phi(A), A) .
$$

Thus we deduce that $d_{H}(\phi(A), A)=0$, which means that $\phi(A)$ is a positive scalar multiple of $A$ for every $A \in B(H)_{-1}^{+}$.

In the case where every nontrivial projection is of type (b), we obtain in a similar manner that $\phi(A)$ is a positive scalar multiple of $A^{-1}$ for every $A \in B(H)_{-1}^{+}$.

Finally, remembering the reductions $\phi(I)=I$ and $U=I$ that we applied in the course of the proof, we see that our original map is of one of the forms appearing in the formulation of the theorem. This completes the proof.

Remark. As the referee remarked, one could possibly reach the result in Theorem 2 also by restricting the considerations from $B(H)_{-1}^{+}$to the set of all elements of unit norm. Indeed, on that set the Hilbert projective metric is a true metric (not only a pseudo-metric). However, we have kept our original approach mainly because the multiplicative structure has seemed easier to handle in that setting.

\section{ACKNOWLEDGEMENTS}

The author is grateful to the referee for comments and advice that have improved the presentation of the paper. In particular, he/she formulated the Lemma for general metric spaces and suggested presenting it as a separate statement. Most of the proof of the Lemma (in the special setting of the space $B(H)_{-1}^{+}$equipped with

the Thompson metric) was originally incorporated in the proof of Theorem 1 .

\section{REFERENCES}

1. T. Ando, Topics on operator inequalities, mimeographed lecture notes, Hokkaido University, Sapporo, 1978. MR0482378 (58:2451)

2. T. Ando, On the arithmetic-geometric-harmonic mean inequality for positive definite matrices, Linear Algebra Appl. 52-53 (1983), 31-37. MR709342 (84j:15016)

3. E. Andruchow, G. Corach and D. Stojanoff, Geometrical significance of the Löwner-Heinz inequality, Proc. Amer. Math. Soc. 128 (2000), 1031-1037. MR1636922 (2000j:46100)

4. G. Birkhoff, Extensions of Jentzsch's theorem, Trans. Amer. Math. Soc. 85 (1957), 219-227. MR 0087058 (19:296a)

5. G. Corach, A. Maestripieri and D. Stojanoff, Orbits of positive operators from a differentiable viewpoint, Positivity 8 (2004), 31-48. MR2053574 (2005d:58011)

6. R.J. Fleming and J.E. Jamison, Isometries on Banach Spaces: Function Spaces, Chapman \& Hall/CRC Monographs and Surveys in Pure and Applied Mathematics, 129, CRC Press, Boca Raton, FL, 2003. MR 1957004 (2004j:46030)

7. R.J. Fleming and J.E. Jamison, Isometries on Banach Spaces: Vector-valued Function Spaces and Operator Spaces, Chapman \& Hall/CRC Monographs and Surveys in Pure and Applied Mathematics, 138, CRC Press, Boca Raton, FL, 2007. MR2361284

8. J. Fujii, Arithmetic-geometric means of operators, Math. Japon. 23 (1979), 667-669. MR529901 (80g:47006)

9. S. Gudder and G. Nagy, Sequentially independent effects, Proc. Amer. Math. Soc. 130 (2002), 1125-1130. MR1873787 (2002i:81014)

10. D. Hilbert, Neue Begaündung der Bolya-Lobatschefskyschen Geometrie, Math. Ann. 57 (1903), 137-150. MR1511203 
11. F. Kubo and T. Ando, Means of positive linear operators, Math. Ann. 246 (1980), 205-224. MR563399 (84d:47028)

12. L. Molnár, Non-linear Jordan triple automorphisms of sets of self-adjoint matrices and operators, Studia Math. 173 (2006), 39-48. MR2204461 (2006j:47059)

13. L. Molnár, Maps preserving the geometric mean of positive operators, Proc. Amer. Math. Soc. 137 (2009), 1763-1770.

14. L. Molnár and P. Šemrl, Non-linear commutativity preserving maps on self-adjoint operators, Quart. J. Math. 56 (2005), 589-595. MR2182468 (2006m:47063)

15. R.D. Nussbaum, Hilbert's projective metric and iterated nonlinear maps, Mem. Amer. Math. Soc. 391 (1988). MR.961211 (89m:47046)

16. R.D. Nussbaum, Finsler structures for the part metric and Hilbert's projective metric and applications to ordinary differential equations, Differ. Integral Equ. 7 (1994), 1649-1707. MR 1269677 (95b:58010)

17. R.D. Nussbaum and J.E. Cohen, The arithmetic-geometric mean and its generalizations for noncommuting linear operators, Ann. Sc. Norm. Super. Pisa 15 (1988), 239-308. MR 1007399 (90e:47018)

18. W. Pusz and S. L. Woronowicz, Functional calculus for sesquilinear forms and the purification map, Rep. Math. Phys. 8 (1975), 159-170. MR0420302 (54:8316)

19. A.C. Thompson, On certain contraction mappings in a partially ordered vector space, Proc. Amer. Math. Soc. 14 (1963), 438-443. MR0149237(26:6727)

20. J. Väisälä, A proof of the Mazur-Ulam theorem, Amer. Math. Monthly 110 (2003), 633-635. MR.2001155 (2004d:46021)

21. A. Vogt, Maps which preserve equality of distance, Studia Math. 45 (1973), 43-48. MR0333676 (48:12001)

Institute of Mathematics, University of Debrecen, P.O. Box 12, H-4010 Debrecen, HUNGARY

E-mail address: molnarl@math.klte.hu

URL: http://www.math.klte.hu/ molnarl/ 